\title{
Adesão ao tratamento da ambliopia
}

\author{
Compliance with amblyopia treatment
}

\author{
Mara Regina Arakaki ${ }^{1}$ \\ Silvana Artioli Schellini ${ }^{2}$ \\ Felipe Jorge Heimbeck ${ }^{3}$ \\ Margareth Tiemi Furuya ${ }^{4}$ \\ Carlos Roberto Padovani ${ }^{5}$
}

\begin{tabular}{|c|}
\hline RESUMO \\
\hline Objetivo: Avaliar a influência da adesão no resultado do tratamento da \\
ambliopia. Métodos: Foi feito estudo retrospectivo avaliando a resposta \\
ao tratamento da ambliopia em 151 crianças portadoras de ambliopia, \\
tratadas com esquema de oclusão diária, com o número de dias variando \\
de acordo com a idade da criança. Os pacientes foram separados em dois \\
grupos que diferiam entre si pela adesão ou não ao tratamento. Resultados: \\
Observou-se que o tratamento teve melhores resultados nas crianças de \\
4 a 7 anos e que aderiram ao tratamento; as crianças que aderiram ao \\
tratamento necessitaram de tempo menor de permanência em tratamento. \\
Mesmo crianças com idade superior a 7 anos tiveram resultados positivos \\
com a oclusão. Conclusão: O tratamento oclusivo é um meio terapêutico \\
eficiente desde que haja a cooperação do paciente.
\end{tabular}

Descritores: Ambliopia/terapia; Curativos oclusivos; Cooperação do paciente; Erros de refração/terapia; Acuidade visual
Faculdade de Medicina de Botucatu - Universidade Estadual Paulista (UNESP) - Depto de OFT/ORL/CCP

Ex-Residente de Oftalmologia.

${ }^{2}$ Professora Livre-docente.

${ }^{3}$ Professor Assistente e Chefe do Setor de Estrabismo da Universidade Estadual Paulista - UNESP.

${ }^{4}$ Ortoptista - Departamento de Oftalmologia, Otorrinolaringologia e Cirurgia de Cabeça e Pescoço da Faculdade de Medicina de Botucatu da Universidade Estadual Paulista - UNESP.

${ }^{5}$ Professor Titular do Departamento de Bioestatística do Instituto de Biociências da Universidade Estadual Paulista - UNESP.

Endereço para correspondência: Silvana Artioli Schellini - Depto de OFT/ORL/CCP - Faculdade de Medicina de Botucatu-UNESP - Botucatu (SP) CEP 18618-000

E-mail:sartioli@fmb.unesp.br

Recebido para publicação em 13.03.2003

Versão revisada recebida em 30.06 .2003

Aprovação em 06.11.2003

\section{INTRODUÇ̃̃̃O}

Ambliopia classicamente significa baixa visão em olho no qual não se detecta alteração orgânica que a justifique ${ }^{(1)}$. Mais recentemente o significado de ambliopia tem se ampliado, significando baixa visão com ou sem lesão orgânica ${ }^{(2)}$. Porém, o assunto é polêmico e esta definição não é aceita por todos.

A mais importante das modalidades terapêuticas empregadas para o tratamento da ambliopia é a oclusão do olho de melhor visão, com a finalidade de estimular o olho com baixa visão, sendo a oclusão considerada o único recurso eficaz para tratamento da ambliopia ${ }^{(3)}$.

A oclusão tem sido o tratamento da ambliopia por cerca de 250 anos, não se tendo nunca avaliado com rigor o seu papel ${ }^{(4)}$.

Esse tipo de tratamento traz bons resultados, na dependência do paciente realizá-lo adequadamente, devendo ser mantido por período variável, conforme a idade da criança sendo, em média, de 3 anos $^{(5)}$, ou mantido até a idade de 10 ou 11 anos, com oclusão de pelo menos uma hora por dia.

Quando existe alteração anatômica, observa-se que a oclusão total do olho diretor também pode ter sucesso, principalmente em casos de opacidade moderada de meios. Em outros casos, como lesão macular ou anomalia de nervo óptico, o prognóstico é pior. Entretanto, no momento, não há melhor tratamento que a oclusão para tratamento da ambliopia ${ }^{(6)}$.

Há indícios de que há melhora da acuidade visual e função binocular nos pacientes submetidos a tratamento oclusivo, sendo que o índice de sucesso varia com a idade do início da terapia, desde que o paciente tenha boa adesão e que seja monitorizado a longo prazo $^{(7)}$.

A adesão rigorosa ao tratamento é tida como decisiva no resultado, de forma que os pacientes que realizam corretamente a oclusão tendem a 
obter acuidade visual final melhor do que os que a realizam irregularmente ${ }^{(8)}$.

Apesar da oclusão ser o tratamento clássico propagado para tratamento da ambliopia, os esquemas utilizados são variáveis, alguns preconizando dias, outros horas de oclusão. No Serviço onde o presente estudo foi desenvolvido, padronizou-se o tratamento constante, o dia todo, com o número de dias baseado na idade da criança por se entender que a resposta ao tratamento é mais adequada desta forma.

O objetivo deste estudo foi conhecer os resultados obtidos com o tratamento empregado em nosso Serviço, avaliando-se quanto à adesão ao tratamento pode influenciar no tratamento da ambliopia.

\section{MÉTODOS}

Realizou-se estudo observacional retrospectivo em crianças menores de 12 anos, atendidas no Ambulatório de Estrabismo da Faculdade de Medicina de Botucatu - UNESP, no período de 1990 até 2000. Dois dos autores (FJH e MTF) acompanharam estas crianças ao longo de todo o período selecionado para estudo.

Os pacientes foram avaliados quanto: sexo, idade de início do tratamento, hipótese diagnóstica e resposta ao tratamento efetuado. As crianças foram divididas em dois grupos, segundo a adesão ou não ao tratamento, avaliando-se a melhora da acuidade visual.

Considerou-se como acuidade visual inicial aquela que a criança informou pela primeira vez, mesmo que o tratamento já houvesse sido instituído há algum tempo. Não fizeram parte deste estudo as crianças não colaborativas ou as que estavam em idade pré-verbal.

O conceito de ambliopia empregado foi diferença de acuidade visual entre um olho e o outro igual ou maior que duas linhas, ou a acuidade visual menor que duas linhas do esperado como normal para a idade.

O esquema de oclusão adotado foi a oclusão durante o dia todo, diretamente na pele, com o número de dias dependente da idade da criança, sendo igual a esta o número de dias de oclusão do olho bom, seguido por um dia de oclusão do olho ruim e assim sucessivamente.

Os resultados foram estatisticamente analisados por meio do Teste de Goodman para contrastes entre e dentro de populações multinominais. A significância está apontada nas tabelas por meio de letras: letras maiúsculas comparam os resultados obtidos dentro de um mesmo grupo de estudo (na horizontal); letras minúsculas comparam resultados entre os grupos (na vertical). A diferença estatística está indicada por letras diferentes, ou seja, letra A é diferente de B, o que indica que houve significância.

\section{RESULTADOS}

No período estudado foram diagnosticadas e tratadas no Serviço de Estrabismo da Faculdade de Medicina de Botucatu
205 crianças portadoras de ambliopia, sendo selecionadas para este estudo 151. As demais foram excluídas por estarem na fase pré-verbal ou por não se ter obtido nos prontuários de estudo as informações necessárias.

Dos pacientes que fizeram parte do estudo $50,6 \%$ eram do sexo masculino. A idade mais freqüente de início do tratamento foi de 4 a 7 anos.

Das crianças estudadas, $85,9 \%$ possuíam estrabismo, $6,6 \%$ anisometropia, $0,7 \%$ catarata congênita e $6,6 \%$ outros diagnósticos (atrofia de papila, placa central de retinocoroidite ou associação de diagnósticos anteriores).

Com relação ao tipo de desvio ocular apresentado, 60,6\% apresentavam esotropia, $13,3 \%$ exotropia, $0,7 \%$ hipertropia, $4,4 \%$ desvio horizontal e vertical e $11,8 \%$ eram ortofóricas (portadoras de anisometropia ou micro-estrabismo).

As crianças que aderiram ao tratamento foram $111 \mathrm{e}$ as que não aderiram foram 40 .

A maioria das crianças que aderiram ao tratamento tiveram ganho de acuidade visual de 1 a 2 linhas (0,532 - Tabela 1). Nos que não aderiram, houve predomínio do ganho de 1 a 2 linhas ou não houve melhora (0,600 - Tabela 1$)$. A comparação de ambos os grupos mostrou diferença estatística no grupo de adesão, com ganho de 3 ou 4 linhas sobre os que não aderiram ao tratamento $(0,270 \times 0,075$ - Tabela 1$)$.

A tabela 2 mostra a acuidade visual com relação ao intervalo de tempo de tratamento para que houvesse melhora. No grupo de adesão ao tratamento, a maioria das crianças levou 6 meses ou mais para ter melhora da visão $(0,432$ - Tabela 2$)$. Nos de não aderiram ao tratamento também houve necessidade de tempo maior que 6 meses para início da melhora visual na maioria das crianças $(0,375$ - Tabela 2). A comparação de ambos os grupos - adesão e não adesão ao tratamento - não mostrou diferença estatística significativa com relação ao tempo de melhora da AV (Tabela 2).

A avaliação do ganho de acuidade visual final em função da adesão ao tratamento mostrou que no grupo em de houve adesão predominou o ganho maior ou igual a 5 linhas $(0,414$ Tabela 3). Nos que não aderiram corretamente ao tratamento, o ganho de acuidade visual foi semelhante nas diversas faixas avaliadas, não havendo diferença estatística significativa (Tabela 3). Comparando os que aderiram ao tratamento com os que não aderiram, houve predomínio significativo do ganho de 5 ou mais linhas no grupo de adesão $(0,414$ X 0,150 - Tabela 3$)$.

Na tabela 4 está apresentado o tempo de manutenção da

\begin{tabular}{|c|c|c|c|c|}
\hline \multirow[b]{2}{*}{ Adesão } & \multicolumn{4}{|c|}{ Ganho de AV } \\
\hline & Nenhum & 1 ou $2 \mathrm{~L}$ & 3 ou $4 \mathrm{~L}$ & $>5 \mathrm{~L}$ \\
\hline Sim & $0,108 \mathrm{aA}$ & $0,532 \mathrm{aC}$ & $0,270 \mathrm{bB}$ & $0,090 \mathrm{aA}$ \\
\hline Não & $0,275 \mathrm{aB}$ & $0,600 \mathrm{aC}$ & 0,075 aA & $0,050 \mathrm{aA}$ \\
\hline
\end{tabular}


oclusão em relação à adesão ou não ao tratamento. Para as crianças que aderiram ao tratamento, houve predomínio da manutenção da oclusão por 1 a 2 anos ou mais. Nos de não adesão, predominou a manutenção por tempo maior que 2 anos (Tabela 4).

Estudando-se o ganho de acuidade visual com relação à idade do início do tratamento, observou-se que nas crianças com início do tratamento entre 4 e 7 anos houve predomínio do ganho de 5 ou mais linhas, seguido por 1 ou 2 linhas e 3 ou 4 linhas; entre os que iniciaram o tratamento com mais de 7 anos, o ganho foi de 3 ou 4 linhas, ou mais de 5 (Tabela 5). Comparando as crianças com relação à idade de início do tratamento, a melhor resposta ao tratamento (mais do que 5 linhas) foi obtida nas crianças que iniciaram o tratamento entre 4 e 7 anos (Tabela 5).

Quanto ao tempo de oclusão, a maioria das crianças com menos de 4 anos ficaram com oclusão por 2 anos ou mais; das com mais que 7 anos, a maioria ficou 1 e 2 anos com oclusão (Tabela 6). A comparação dos grupos entre si mostrou que o tratamento durou menos que 1 ano igualmente acima dos 4 até os 7 anos e acima dos 7 anos, entre 1 e 2 anos principalmente nos de mais de 7 anos e mais que 2 anos principalmente em menores do que 4 anos (Tabela 6).

\section{DISCUSSÃO}

\begin{tabular}{|c|c|c|c|c|}
\hline \multicolumn{5}{|c|}{$\begin{array}{l}\text { Tabela 2. Distribuição do intervalo de tempo para melhora da } \\
\text { acuidade visual inicial em função da adesão ao tratamento }\end{array}$} \\
\hline \multirow[b]{2}{*}{ Adesão } & \multicolumn{4}{|c|}{ Intervalo } \\
\hline & $1-3 m$ & $3-6 m$ & $>6 m$ & SM \\
\hline Sim & $0,027 \mathrm{aA} \quad 0,261 \mathrm{aC}$ & $0,171 \mathrm{aBC}$ & 0,432 aD & $0,108 \mathrm{aAB}$ \\
\hline Não & aA $0,200 a B$ & $0,150 \mathrm{aB}$ & $0,375 \mathrm{aC} 2$ & $0,275 \mathrm{aB}$ \\
\hline \multicolumn{5}{|c|}{$\begin{array}{l}\text { Letras maiúsculas comparam as variáveis na horizontal; letras minúsculas } \\
\text { comparam as variáveis na vertical. Duas letras diferentes indicam significância } \\
\text { estatística; } m=\text { meses; } S M=s e m \text { melhora }\end{array}$} \\
\hline
\end{tabular}

Tabela 3. Distribuição do ganho de acuidade visual final em função da adesão ao tratamento

\begin{tabular}{|lcccc|} 
& \multicolumn{5}{c}{ Ganho de AV } \\
\cline { 2 - 5 } Adesão & Nenhum & $\mathbf{1}$ ou $\mathbf{2} \mathbf{~ L}$ & $\mathbf{3}$ ou $\mathbf{4} \mathbf{~ L}$ & $>\mathbf{5} \mathbf{~}$ \\
Sim & $0,108 \mathrm{aA}$ & $0,225 \mathrm{aA}$ & $0,252 \mathrm{aA}$ & $0,414 \mathrm{bB}$ \\
Não & $0,275 \mathrm{aA}$ & $0,250 \mathrm{aA}$ & $0,325 \mathrm{aA}$ & $0,150 \mathrm{aA}$ \\
Letras maiúsculas comparam as variáveis na horizontal; letras minúsculas \\
comparam as variáveis na vertical. Duas letras diferentes indicam significância \\
estatística; L= linha na Tabela de Snellen
\end{tabular}

\begin{tabular}{|l|lcc|}
\hline \multicolumn{4}{|c|}{$\begin{array}{l}\text { Tabela 4. Distribuição do intervalo de tempo de oclusão em função } \\
\text { da adesão ao tratamento } \\
\text { Intervalo }\end{array}$} \\
\cline { 2 - 4 } Adesão & \multicolumn{4}{c}{$\begin{array}{c}\text { < ano } \\
\text { Sim }\end{array}$} & $\mathbf{1 - 2}$ anos & $>2$ anos \\
Não & $0,189 \mathrm{aA}$ & $0,378 \mathrm{aB}$ & $0,432 \mathrm{aB}$ \\
$\begin{array}{l}\text { Letras maiúsculas comparam as variáveis na horizontal; letras minúsculas } \\
\text { comparam as variáveis na vertical. Duas letras diferentes indicam significância } \\
\text { estatística }\end{array}$ \\
\hline
\end{tabular}

O estudo da ambliopia é difícil de ser feito, uma vez que o próprio conceito de ambliopia não é uniforme. O diagnóstico de que um olho é amblíope é dado segundo a acuidade visual. Porém, não há um consenso sobre qual é a acuidade visual que deve ser encarada como marco para definir a presença da ambliopia. Assim, para alguns existe ambliopia quando a acuidade visual corrigida com o uso da melhor correção óptica é menor que 0,7 ou existe uma diferença entre um olho e outro de 3 linhas ou mais; para outros, a visão menor que 0,4 ; ou, ainda, diferença de duas linhas ou mais entre os dois olhos ${ }^{(9)}$.

As diferentes conceituações dificultam a comparação entre os estudos.

O que mais se conhece sobre a ambliopia são os índices do problema detectado na população em campanhas populacionais, citando-se que a ambliopia acomete cerca de 0,77 a 4\% das crianças $^{(9-12)}$.

A maior parte das crianças deste estudo era portadora de ambliopia estrabísmica, sendo esta uma causa freqüente de ambliopia. As principais causas de ambliopia na infância são as ametropias e o estrabismo ${ }^{(13)}$. Mas a detecção do problema não é garantia de cura da ambliopia, cujo tratamento, em geral, é longo, requerendo adesão e persistência dos pais e da criança.

Neste sentido, é muito importante que sejam divulgados os estudos de séries de pacientes para que se possa melhor balizar o acompanhamento.

A maioria (41,8\%) das crianças que fizeram parte do presente estudo apresentavam idade entre 4 a 7 anos. Mas gostaríamos de ressaltar que $29,1 \%$ das crianças apresentavam mais de 7 anos e não haviam anteriormente feito qualquer tratamento prévio quando foi iniciado o tratamento. Classicamente, a acuidade visual se desenvolve a partir do nascimento e a crian-

Tabela 5. Distribuição do ganho de acuidade visual total em função da idade de início do tratamento

\begin{tabular}{lcccc} 
& \multicolumn{5}{c}{ Ganho de AV } \\
\cline { 2 - 5 } Idade & Nenhum & $\mathbf{1}$ ou $\mathbf{2}$ L & $\mathbf{3}$ ou $\mathbf{4} \mathrm{L}$ & $>\mathbf{5 L}$ \\
$\mathbf{0 - 4}$ anos & $0,283 \mathrm{bA}$ & $0,304 \mathrm{aA}$ & $0,239 \mathrm{aA}$ & $0,174 \mathrm{aA}$ \\
$\mathbf{4 - 7}$ anos & $0,076 \mathrm{aA}$ & $0,227 \mathrm{aB}$ & $0,212 \mathrm{aB}$ & $0,485 \mathrm{bC}$ \\
$>\mathbf{7}$ anos & $0,109 \mathrm{abA}$ & $0,174 \mathrm{aA}$ & $0,370 \mathrm{aB}$ & $0,348 \mathrm{bB}$
\end{tabular}

Letras maiúsculas comparam as variáveis na horizontal; letras minúsculas comparam as variáveis na vertical. Duas letras diferentes indicam significância estatística; L= linha na Tabela de Snellen

Tabela 6. Distribuição do intervalo de tempo de oclusão em função da idade de início do tratamento

\begin{tabular}{|c|c|c|c|}
\hline \multirow[b]{2}{*}{ Idade } & \multicolumn{3}{|c|}{ Intervalo } \\
\hline & $<1$ ano & $1-2$ anos & $>2$ anos \\
\hline 0 - 4 anos & 0,065 aA & 0,130 aA & 0,804 bB \\
\hline $4-7$ anos & $0,348 \mathrm{bA}$ & $0,333 \mathrm{bA}$ & $0,318 \mathrm{aA}$ \\
\hline$>7$ anos & $0,196 \mathrm{bA}$ & $0,565 \mathrm{cB}$ & $0,239 \mathrm{aA}$ \\
\hline \multicolumn{4}{|c|}{$\begin{array}{l}\text { Letras maiúsculas comparam as variáveis na horizontal; letras minúsculas } \\
\text { comparam as variáveis na vertical. Duas letras diferentes indicam significância } \\
\text { estatística. }\end{array}$} \\
\hline
\end{tabular}


ça deve ganhar visão até por volta dos 7 anos de idade, época em que se completa o desenvolvimento visual na maioria das crianças. Mas pode existir, ainda depois disso, ganho de acuidade visual, conforme nos foi possível observar. Baseados nisso, acreditamos ser útil sempre tentar o tratamento porque pode existir a chance de ganho. A oclusão realizada em 100 crianças amblíopes, com idade entre 7 e 12 anos, resultou em ganho para cerca de $68 \%$ delas $^{(14)}$, o que reforça a nossa idéia.

Além da idade da criança, o resultado do tratamento pode também ser influenciado pela causa da ambliopia ${ }^{(15)}$, sendo a oclusão mais efetiva nos portadores de ambliopia estrabís$\operatorname{mica}^{(16)}$.

Também a adesão ao tratamento está relacionada com o grau de ambliopia, sendo pior o resultado nas crianças que possuem ambliopia mais grave ${ }^{(8)}$.

Mas, parece que o principal fator que influencia o resultado do tratamento é a acuidade visual inicial ${ }^{(17)}$.

Das crianças que foram por nós estudadas, a maioria (cerca de $73 \%$ ) teve boa adesão ao tratamento. Os estudos a respeito do ganho de acuidade visual segundo a idade ou segundo a adesão ao tratamento são escassos. Sabe-se que a adesão ao tratamento da ambliopia, observada em 496 indivíduos, mostrou que $18 \%$ não realizaram adequadamente o tratamento ${ }^{(8)}$, dado semelhante ao aqui obtido.

A avaliação estatística do ganho visual com relação à adesão ao tratamento mostrou que há correlação positiva entre se fazer adequadamente o tratamento e o ganho de acuidade visual. Crianças que fizeram corretamente a oclusão tiveram ganho de acuidade visual superior as que não fizeram. $\mathrm{O}$ fator crítico para o bom desenvolvimento da visão é a adequada oclusão do olho bom, além da adequada correção do erro refracional $^{(18)}$.

A avaliação do tempo de tratamento para melhora mostrou que, mesmo que haja colaboração, há necessidade de tempo prolongado para se obter melhora, já havendo sido previamente relatado tempo de 6 meses para os efeitos da oclusão serem sentidos ${ }^{(16)}$.

O ganho de acuidade visual foi quantitativamente maior nas crianças que realizaram corretamente a oclusão, podendo se ter aumento de até 5 linhas de melhora da acuidade visual.

Outra observação importante foi que o tempo de manutenção da oclusão foi longo (de 1 a 2 anos) nas crianças que aderiram ao tratamento. Porém, nas que não aderiram, o tempo de manutenção da oclusão foi ainda maior.

A comparação do tempo de manutenção da oclusão com relação à idade mostrou tempo semelhante em crianças menores e maiores. Este dado não deve ser demasiadamente valorizado, uma vez que nas crianças maiores de 7 anos, quando a oclusão não está surtindo efeito, não manutenção do tratamento pode significar desistência do tratamento e não suspensão por melhora visual.

O tratamento da ambliopia continua sendo um desafio. Mesmo quando se segue corretamente o tratamento, o olho pode continuar amblíope, persistindo com baixa visão 1,9\% dos portadores na idade adulta ${ }^{(11)}$.
Além disso, o tratamento pode resultar em danos para o olho bom. Existem relatos de que ambos os olhos podem ficar com redução da sensibilidade ao contraste ${ }^{(19)}$.

Muitos são os fatores determinantes da adesão ao tratamento, tais como, a idade, o nível de entendimento do problema por parte dos pais, a acuidade visual inicial e a velocidade do ganho de acuidade ${ }^{(8)}$ e todos estes pontos devem ser valorizados para se ter resultados positivos com o tratamento.

De qualquer forma, a melhor prevenção da ambliopia é a sua detecção precoce, o que é feito, em geral, em levantamentos populacionais ${ }^{(20)}$, associada à compreensão do problema, o que vai levar à melhor adesão ao tratamento.

\section{CONCLUSÃO}

Nossos resultados nos permitem concluir que o tratamento da ambliopia é, em geral, longo. A melhora da acuidade visual pode ocorrer mesmo em crianças com idade superior a 7 anos, sendo que a adesão ao tratamento é fundamental para que se tenha um ganho significativo de acuidade visual.

\section{ABSTRACT}

Purpose: To evaluate results of compliance with amblyopia treatment. Methods: A retrospective study was performed to evaluate the influence of compliance with amblyopia treatment in 151 children, treated with all-day occlusion, which was variable according to the childrens' age. The patients were divided in to two groups according to compliance or not with the treatment. Results: The treatment presented best results in children 4 and 7 years of age and in children with a good compliance. These children took less time to be treated. Even children of 7 years or more had good results with the treatment. Conclusion: Occlusion is a very good treatment when the patients comply with it.

Keywords: Amblyopia/therapy; Occlusive dressings; Patient compliance; Refractive errors/therapy; Visual acuity

\section{AGRADECIMENTO}

Agradecemos ao doutor Marcelo Rocco que nos auxiliou na coleta de dados para a realização deste estudo.

\section{REFERÊNCIAS}

1. Hugonnier RS. Estrabismos. Barcelona: Toray-Masson; 1977.

2. Diaz JP, Dias CS. Estrabismo. São Paulo: Rocca; 1986.

3. Endo RM. Treatment of amblyopia. An Oftalmol 1988;7:45-7.

4. Fielder AR, Irwin M, Auld R, Cocker KD, Jones HS, Moseley MJ. Compliance in amblyopia therapy: objective monitoring of occlusion. $\mathrm{Br} \mathrm{J}$ Ophthalmol 1995;79:585-9.

5. Oster JG, Simon JW, Jenkins P. When is it safe to stop patching? Br J Ophthalmol 1990;74:709-11. 
6. Bradfort GM, Kutsher PJ, Scott WE. Results of amblyopia therapy in eyes with unilateral structural abnormalities. Ophthalmology 1992;99:1616-21.

7. Wick B, Wingard M, Cotter S, Scheiman M. Anisometropic amblyopia: is the patient ever too old to treat? Optom Vis Sci 1992;69:866-78.

8. Nucci P, Alfarano R, Piantanida A, Brancato R. Compliance in anti-amblyopia occlusion therapy.Acta Ophthalmol 1992;70:128-31.

9. Schellini SA, Shimoda GA, Moraes-Silva MRB, Utyama LC, Isano C. Prevalência de alterações oculares em pré-escolares em Botucatu-SP. Rev Bras Oftalmol 1987;47:34-7.

10. Kara-Jose N, Carvalho KM, Caldato RP, Oliveira VL, Fonseca-Neto AM, Correa J. Amblyopia care and prevalence among preschool children, Campinas, São Paulo Brazil. Bol Oficina Sanit Panam 1984;96:31-7.

11. Thompson JR, Woodruff G, Hiscox FA, Strong N, Minshull C. The incidence and prevalence of amblyopia detected in childhood. Public Health 1991; 105:455-62.

12. Latvala ML, Paloheimo M, Karma A. Screening of amblyopic children and long-term follow-up. Acta Ophthalmol Scand 1996;74:488-92.

13. Correal PM. Transtornos de la agudeza visual en la edad escolar. Visual acuity disorders in childhood. 1982. S.1.: S.d. 130p.
14. Oréfice NL. Tratamento da ambliopia em crianças com idade acima dos 7 anos. Rev Bras Oftalmol 1992;51:387-90.

15. Simmers A J, Gray LS, McGraw PV, Winn B. Functional visual loss in amblyopia and the effect of occlusion therapy. Invest Ophthalmol Vis Sci 1999:40:2859-71.

16. Cleary M. Efficacy of occlusion for strabismic amblyopia: Can an optimal duraction be identified. Br J Ophthalmol 2000;84:572-8

17. Woodruff G, Hiscox F, Thompson JR, Smith LK. Factors affecting the outcome of children treated for amblyopia[comment on Eye 1995;9(Pt 5):664]. Eye1991;8(Pt 6):627-31

18. Elsas FJ. Visual acuity in monocular pediatric aphakia: does epikeratophakia facilitate occlusion therapy in children intolerant of contact lens or spectacle wear. J Pediatr Ophthalmol Strabimus 1990;27:304-9.

19. Wali N, Leguire LE, Rogers GL, Bremer DL. Csf interocular interactions in childhood amblyopia. Optom Vis Sci 1991;68:81-7.

20. Flynn JT, Schiffman J, Feuer W, Corona A. The therapy of amblyopia: an analysis of the results of amblyopia therapy utilizing the pooled data of published studies. Trans Am Ophthalmol Soc 1998;96:431-50; discussion p.450-3.

\section{CONGRESSO DA SOCIEDADE CAIPIRA DE OFTALMOLOGIA}

\section{2 e 3 de julho de 2004 \\ Centro de Convenções \\ do Hospital das Clínicas de \\ Ribeirão Preto - SP}

\section{Informações e Inscrições: CENACON \\ Tel.: (17) 235-7017/Fax: (17) 235-5334 \\ E-mail: cenacon@cenacon.com.br \\ Home-page: www.cenacon.com.br}

